\section{Efficacy of Periapical Radiography and Three Cone-Beam Computed Tomography Systems for Detection of Peri-Implant Dehiscence Defects: An in- Vitro Study}

\author{
Akheshteh V. ${ }^{1 \oplus}$, Eskandarloo A. ${ }^{2 * \odot}$, Saati S.3, Jamalpour M. \\ R. ${ }^{4}$, Mohammad Gholi Mezerji N. ${ }^{5}$
}

\begin{abstract}
Background: Early detection of peri-implant bone defects is highly important because these defects eventually lead to gingival recession, bone loss and implant failure.
\end{abstract}

Objective: This study aimed to assess and compare the efficacy of periapical radiography and three $\mathrm{CBCT}$ systems for the detection of peri-implant dehiscence defects.

Material and Methods: In this vitro study, 124 titanium implants were placed in bovine ribs. The bone pieces were then mounted in boxes in the form of mandible and red dental wax was used to simulate the soft tissue. Crestal bone defects with 2, 3 , and $4 \mathrm{~mm}$ depth were created in the ribs using a round bur. Periapical and CBCT images were then obtained. Images were investigated by two oral and maxillofacial radiologists twice with a two-week interval. The results were analyzed using chisquare, Kappa coefficient, Cochrane's Q and McNemar tests as well as the receiver operating characteristic (ROC) curve.

Results: The two observers showed good agreement in detection of sound and defective samples on periapical radiographs and CBCT scans. The level of agreement was low in detection of two samples with $2 \mathrm{~mm}$ defects on CBCT scans taken with Planmeca and NewTom 3G systems at the time of second assessment. NewTom 3G had the highest sensitivity $(68.9 \%, 74.2 \%$ and $86.3 \%$, respectively) and specificity ( $100 \%$ for all three) compared to other systems for detection of 2,3 and $4 \mathrm{~mm}$ crestal bone defects.

Conclusion: The inter-observer agreement increased with increase in depth of defects. NewTom $3 \mathrm{G}$ had the highest accuracy for detection of crestal bone defects.

Citation: Akheshteh V, Eskandarloo A, Saati S, Jamalpour MR, Mohammad Gholi Mezerji N. Efficacy of Periapical Radiography and Three Cone-Beam Computed Tomography Systems for Detection of Peri-Implant Dehiscence Defects: An in- Vitro Study. J Biomed Phys Eng. 2020;10(6):751-760. doi: 10.31661/jbpe.v0i0.2008-1162.

\section{Keywords}

Surgical Wound Dehiscence; Cone Beam Computed Tomography; Radiography, Dental

\section{Introduction}

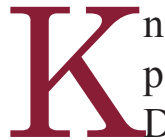

nowledge about the presence of cortical plate around dental implants is imperative for success of dental implant treatment [1]. rect anatomical location or positioning of implant, excessive load ap-
${ }^{1} \mathrm{MSD}$, Department of Oral and Maxillofacial Radiology, Alborz University of Medical Sciences,

Tehran, Iran

${ }^{2} \mathrm{MSD}$, Department of

Oral and Maxillofacial

Radiology, School of

Dentistry, Hamadan

University of Medical Sci-

ences, Hamedan, Iran

${ }^{3} \mathrm{MSD}$, Department of

Oral and Maxillofacial

Radiology, Hamadan

University of Medical Sci-

ences, Hamedan, Iran

${ }^{4} \mathrm{MSD}$, Department of

Oral and Maxillofacial

Surgery, Hamadan

University of Medical Sci-

ences, Hamedan, Iran

${ }^{5} \mathrm{PhD}$, Department of

Biostatistics, School of

Public Health, Hamadan

University of Medical Sci-

ences, Hamedan, Iran

*Corresponding author:

A. Eskandarloo

Department of Oral and

Maxillofacial Radiology,

School of Dentistry,

Hamadan University

of Medical Sciences,

Hamedan, Iran

E-mail: dr.eskandarloo@ gmail.com

Received: 17 August 2020

Accepted: 14 September 2020 
plied to implants or inflammation caused by the biofilm. These defects prevent complete coverage of implant surface and cause hygienic and esthetic problems. Therefore, early detection of peri-implant bone defects is highly important because these defects eventually lead to gingival recession, bone loss and implant failure [2-4].

Postoperative radiographic assessment of marginal bone loss around dental implants is pivotal in this respect [5-9]. Diagnostic imaging is used as the leading technique for assessment of alveolar bone height and detection of bone defects [10]. Radiographic modalities used for evaluation of bone defects include conventional and digital intraoral radiography, panoramic radiography, computed tomography (CT) and cone-beam computed tomography (CBCT) [11].

Intraoral periapical radiography with the long cone paralleling technique is commonly used for assessment of dental implants. This imaging modality has low patient radiation dose, low cost and high resolution and can be used chairside. This technique has sufficient accuracy for postsurgical assessment of dental implants. However, it is not suitable for detection of crestal bone loss in the buccal and lingual plates around dental implants due to the two-dimensional (2D) nature of images and superimposition of anatomical structures. This modality is only suitable for evaluation of interproximal bone $[2,10,11]$.

More advanced imaging techniques such as $\mathrm{CT}$ provide more accurate information in all three dimensions. However, their application for dental purposes is limited due to their high cost, large size of equipment and high patient radiation dose [12]. CBCT is currently used as a standard alternative for many diagnostic procedures [13]. Moreover, CBCT images have high accuracy and quality and provide $3 \mathrm{D}$ views with no distortion, allowing precise assessment of bone defects in all three dimensions [2, 11]. Also, considering the recent advances in CBCT systems and their differ- ent image reconstruction capabilities, fields of view (FOVs) and software programs, it is important to find the most efficient CBCT system to ideally visualize the cortical plates and bone defects on 3D images. Considering the significance of this topic, this study aimed to assess and compare the efficacy of NewTom 3G, Planmeca(Promax 3D) and Soredex (Cranex3D)CBCT systems and periapical radiography for detection of dehiscence around dental implants.

\section{Material and Methods}

\section{Preparation of samples}

In this vitro study, fresh bovine ribs were used to simulate the jawbone and two layers of red dental wax were applied to simulate the soft tissue. The ribs were cut into pieces using a saw for mounting in boxes simulating the mandible. Titanium implants $(n=124)$ measuring $11 \times 4.5 \mathrm{~mm}$ (SIC Invent AG, Switzerland) were inserted in the ribs by an expert oral and maxillofacial surgeon. The buccal cortical plate was then removed using a trimmer (Doppelschoiben-Modelltrimmer, Dentaurum, Germany) such that the distance from the implant to the reference line in the buccal surface was $2 \mathrm{~mm}$.

\section{Imaging modalities}

Periapical radiographs were obtained with digital intraoral X-ray unit (Minray; Soredex, Tuusula, Finland) and the exposure settings of $7 \mathrm{~mA}, 0.25 \mathrm{~s}$ and $60 \mathrm{kVp}$ using the paralleling technique and size 2 photostimulable phosphor plate (PSP) detector. To maintain the geometry and have reproducibility, an intraoral film holder was adjusted at $5 \mathrm{~mm}$ distance from the healing abutment screwed into the implant. The X-ray tube was fixed to the film holder using putty impression material.

CBCT images were taken using Cranex 3D (Soredex, Tuusula, Finland) with the exposure settings of 6-inch FOV, $4 \mathrm{~mA}, 6.1 \mathrm{~s}$ and 110 kVp, NewTom 3G (Quantitative Radiology, 
Verona, Italy) with the exposure settings of 6-inch FOV, $10.65 \mathrm{~mA}, 110 \mathrm{kVp}$ and Promax 3D (Planmeca, Helsinki, Finland) with the exposure settings of 8-inch FOV, $14 \mathrm{~mA}, 12 \mathrm{~s}$ and $84 \mathrm{kVp}$.

\section{Creation of crestal bone resorp-} tion defects

After obtaining control (baseline) images, the crestal bone was incrementally removed in a semilunar fashion using a round bur (1/2 and 1/4, Green; Teezkavan, Iran) such that the created defects resembled natural defects and were extended beyond the mesial and distal margins of implants by $0.5 \mathrm{~mm}$. A total of 124 defects (31 samples with $2 \mathrm{~mm}$ deep dehiscence defects, 31 samples with $3 \mathrm{~mm}$ deep dehiscence defects and 31 samples with $4 \mathrm{~mm}$ deep dehiscence defects) were created as such. CBCT scans (with the three systems) and periapical radiographs were taken again as explained earlier (Figures 1 and 2).

Cross-sectional slices with $1 \mathrm{~mm}$ slice thickness and $1 \mathrm{~mm}$ interval were reconstructed of each inserted implant such that the middle section was made at the site of the highest implant diameter. Two experienced oral and maxillofacial radiologists inspected all im-
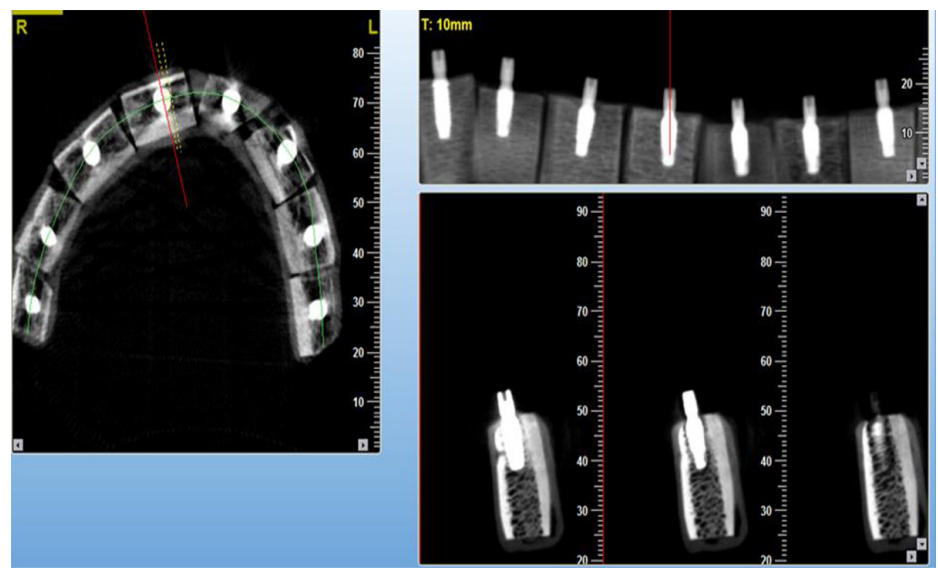

Figure 1: Cone-beam computed tomography (CBCT) image of crestal bone defects ( $3 \mathrm{~mm}$ depth, Newtom 3G)

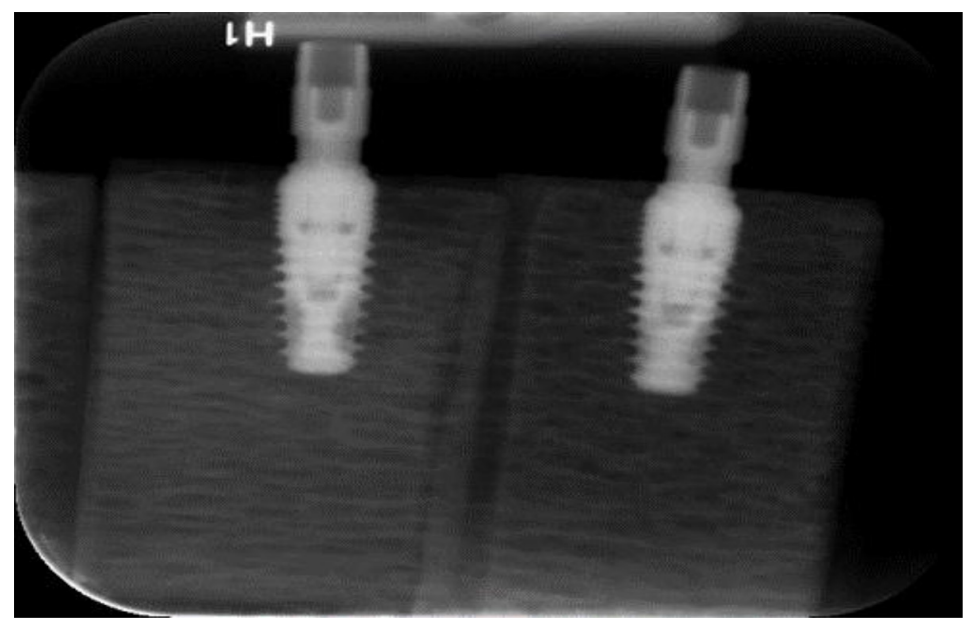

Figure 2: Periapical image of crestal bone defects ( $3 \mathrm{~mm}$ depth) 
Akheshteh V., Eskandarloo A., Saati S. et al

ages blindly on a 20-inch monitor (LG-200p; LG, Seoul, Korea) in a semi-dark room under similar conditions. They were allowed to adjust the brightness and contrast of images and used magnification (zoom feature). The two observers recorded their observations regarding presence/absence of defects in a checklist. Evaluation was repeated in a 2 -weeks interval. Sensitivity and specificity values were calculated for each imaging modality and compared among different modalities. The Cohen's kappa statistic was calculated to assess the intraand inter-observer agreements. The kappa values were interpreted according to the Landis and Koch classification modified by Altman as follows: $\mathrm{k} \leq 0.2$ : poor, $0.21-0.40$ : fair, 0.41 0.60: moderate, 0.61-0.80: good, 0.81-1.0: very good. The Cochrane's Q test was used to compare the four radiographic modalities. Data were analyzed using SPSS version 16 (SPSS Inc., IL, USA). Alpha equal to 0.05 was considered in all tests.

\section{Results}

In most cases, the intra-observer agreement was $>70 \%$, translating to very good except for detection of two samples with $2 \mathrm{~mm}$ defects on CBCT scans taken by Planmeca and NewTom CBCT systems (Table 1).

As shown in Table 2, the two observers had good agreement in detection of sound and defective samples in use of all CBCT systems except for two samples with $2 \mathrm{~mm}$ defects on CBCT scans taken with Planmeca and NewTom systems at the second observation time, for which, the two observers had low agree-

Table 1: Intra-observer agreement according to the imaging modality and depth of defect using Cohen's kappa coefficient.

\begin{tabular}{cccccc} 
& Defect & Planmeca & Cranex 3D & NewTom & PSP \\
\hline & Sound & $1.0(\mathrm{P}<0.001)$ & $1.0(\mathrm{P}<0.001)$ & $1.0(\mathrm{P}<0.001)$ & $1.0(\mathrm{P}<0.001)$ \\
\cline { 2 - 6 } Rater 1 & $2 \mathrm{~mm}$ & $0.803(\mathrm{P}<0.001)$ & $0.864(\mathrm{P}<0.001)$ & $0.912(\mathrm{P}<0.001)$ & $1.0(\mathrm{P}<0.001)$ \\
\cline { 2 - 6 } & $3 \mathrm{~mm}$ & $0.871(\mathrm{P}<0.001)$ & $1.0(\mathrm{P}<0.001)$ & $1.0(\mathrm{P}<0.001)$ & $1.0(\mathrm{P}<0.001)$ \\
\cline { 2 - 6 } & $4 \mathrm{~mm}$ & $1.0(\mathrm{P}<0.001)$ & $0.815(\mathrm{P}<0.001)$ & $0.870(\mathrm{P}<0.001)$ & $1.0(\mathrm{P}<0.001)$ \\
\hline \multirow{3}{*}{ Rater 2 } & Sound & $1.0(\mathrm{P}<0.001)$ & $1.0(\mathrm{P}<0.001)$ & $1.0(\mathrm{P}<0.001)$ & $1.0(\mathrm{P}<0.001)$ \\
\cline { 2 - 6 } & $2 \mathrm{~mm}$ & $-0.1 \mathrm{P}=0.576$ & $0.934(\mathrm{P}<0.001)$ & $0.295 \mathrm{P}=0.1$ & $1.0(\mathrm{P}<0.001)$ \\
\cline { 2 - 6 } & $3 \mathrm{~mm}$ & $1.0(\mathrm{P}<0.001)$ & $0.928(\mathrm{P}<0.001)$ & $0.844(\mathrm{P}<0.001)$ & $1.0(\mathrm{P}<0.001)$ \\
\hline & $4 \mathrm{~mm}$ & $0.928(\mathrm{P}<0.001)$ & $0.912(\mathrm{P}<0.001)$ & $0.713(\mathrm{P}<0.001)$ & $0.652(\mathrm{P}<0.001)$
\end{tabular}

Table 2: Inter-observer agreement according to the system used and time of assessment using Cohen's kappa coefficient.

\begin{tabular}{cccccc} 
& & Planmeca & Soredex & NewTom & PSP \\
\hline & Sound & $1.0(\mathrm{P}<0.001)$ & $1.0(\mathrm{P}<0.001)$ & $1.0(\mathrm{P}<0.001)$ & $1.0(\mathrm{P}<0.001)$ \\
\cline { 2 - 6 } Time 1 & $2 \mathrm{~mm}$ & $0.803(\mathrm{P}<0.001)$ & $0.736(\mathrm{P}<0.001)$ & $0.775(\mathrm{P}<0.001)$ & $1.0(\mathrm{P}<0.001)$ \\
\cline { 2 - 6 } & $3 \mathrm{~mm}$ & $0.807(\mathrm{P}<0.001)$ & $0.931(\mathrm{P}<0.001)$ & $0.912(\mathrm{P}<0.001)$ & $1.0(\mathrm{P}<0.001)$ \\
\cline { 2 - 6 } & $4 \mathrm{~mm}$ & $0.793(\mathrm{P}<0.001)$ & $0.912(\mathrm{P}<0.001)$ & $1.0(\mathrm{P}<0.001)$ & $1.0(\mathrm{P}<0.001)$ \\
\hline \multirow{3}{*}{ Time 2 } & Sound & $1.0(\mathrm{P}<0.001)$ & $1.0(\mathrm{P}<0.001)$ & $1.0(\mathrm{P}<0.001)$ & $1.0(\mathrm{P}<0.001)$ \\
\cline { 2 - 6 } & $2 \mathrm{~mm}$ & $0.159(\mathrm{P}=0.319)$ & $0.799(\mathrm{P}<0.001)$ & $0.233 \mathrm{P}=0.173$ & $1.0(\mathrm{P}<0.001)$ \\
\cline { 2 - 6 } & $3 \mathrm{~mm}$ & $0.806(\mathrm{P}<0.001)$ & $0.860(\mathrm{P}<0.001)$ & $0.760(\mathrm{P}<0.001)$ & $1.0(\mathrm{P}<0.001)$ \\
\hline & $4 \mathrm{~mm}$ & $0.860(\mathrm{P}<0.001)$ & $0.815(\mathrm{P}<0.001)$ & $0.870(\mathrm{P}<0.001)$ & $0.652(\mathrm{P}<0.001)$
\end{tabular}


ment. Complete agreement exists between the two observers at the first and second assessment time points for detection of sound samples.

In general, by an increase in depth of defects, the inter-observer agreement increased. By an increase in depth of defects, the inter-observer agreement on detection of defects on CBCT scans taken with NewTom system increased.

Using Cochrane's Q test, the opinions of the two observers at the two assessment time points regarding presence/absence of defects on images taken with different systems were compared in the four groups. The results showed no significant difference among the four systems in detection of sound samples $(\mathrm{P}=0.3)$. However, the four imaging systems were significantly different in detection of samples with 2, 3 and $4 \mathrm{~mm}$ defects $(\mathrm{P}<0.001)$, (Table 3).

The area under the receiver operating characteristic (ROC) curve was calculated to evaluate sensitivity and specificity at the same time, and indicated higher diagnostic accuracy of NewTom than that of other modalities (Figure 3).

For detection of $2 \mathrm{~mm}$ crestal bone defects, the sensitivity of NewTom (68.9\%) was higher than that of Soredex $(58.9 \%)$, Planmeca $(43.4 \%)$ and periapical radiography $(4.8 \%)$. Also, the specificity of NewTom (100\%) was higher than that of other modalities (Table 4).

The area under the ROC curve was calculated to evaluate sensitivity and specificity at the same time, and indicated higher diagnostic accuracy of NewTom than that of other modalities (Figure 4).

For detection of $3 \mathrm{~mm}$ crestal bone defects, the sensitivity of NewTom (74.2\%) was higher than that of Soredex $(62.7 \%)$, Planmeca $(50.7 \%)$ and periapical radiography $(4.8 \%)$. Also, the specificity of NewTom (100\%) was higher than that of other modalities (Table 4).

The area under the ROC curve was calculated to evaluate sensitivity and specificity at the same time, and indicated higher diagnostic accuracy of NewTom than that of other modalities (Figure 5).

For detection of $4 \mathrm{~mm}$ crestal bone defects, the sensitivity of NewTom $(86.3 \%)$ was higher than that of Soredex $(76.6 \%)$, Planmeca $(63.7 \%)$ and periapical radiography $(7.2 \%)$. Also, the specificity of NewTom $(100 \%)$ was higher than that of other modalities (Table 4).

\section{Discussion}

Radiography is an inseparable part of detection and diagnosis of cortical bone defects. There are several CBCT systems currently available in the market with different image qualities. Considering the lack of studies comparing Cranex 3D, NewTom 3G and Planmeca CBCT systems for detection of crestal bone defects around dental implants, this study compared the efficacy of the afore-mentioned three CBCT systems with periapical radiography for this purpose. Comparison of CBCT systems and periapical radiography revealed higher sensitivity and area under the ROC curve in all three CBCT systems for detec-

Table 3: Efficacy of the four imaging systems for detection of sound and defective samples.

\section{Defects}

\begin{tabular}{ccccccccccc}
\cline { 3 - 10 } & \multicolumn{2}{c}{ Sound } & \multicolumn{2}{c}{$\mathbf{2} \mathbf{~ m m}$} & \multicolumn{2}{c}{$3 \mathbf{~ m m}$} & \multicolumn{2}{c}{$\mathbf{4} \mathbf{~ m m}$} \\
\cline { 3 - 10 } & No & Yes & No & Yes & No & Yes & No & Yes \\
\hline \multirow{5}{*}{ Device } & Planmeca & 116 & 8 & 68 & 56 & 60 & 64 & 45 & 79 \\
\cline { 2 - 10 } & Soredex & 120 & 4 & 51 & 73 & 45 & 79 & 29 & 95 \\
\cline { 2 - 10 } & NewTom & 124 & 0 & 37 & 87 & 32 & 92 & 17 & 107 \\
\cline { 2 - 10 } & PSP & 124 & 0 & 120 & 4 & 120 & 4 & 117 & 7
\end{tabular}




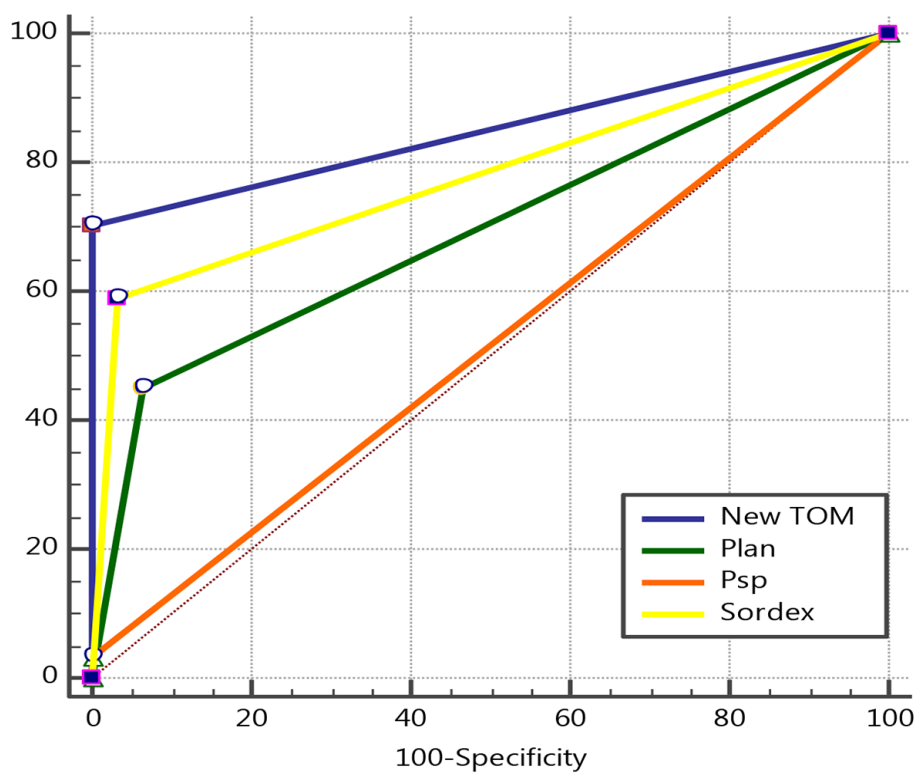

Figure 3: The receiver operating characteristic (ROC) curve for detection of $2 \mathrm{~mm}$ defects using the four imaging modalities.

Table 4: Sensitivity, specificity, false positive and false negative values of the four imaging modalities for detection of 2, 3, $4 \mathrm{~mm}$ deep crestal bone defects.

\begin{tabular}{|c|c|c|c|c|c|c|c|c|}
\hline $\begin{array}{l}\text { Imaging } \\
\text { modality }\end{array}$ & & $\begin{array}{c}\text { Sensitivity } \\
(\%)\end{array}$ & $\begin{array}{c}\text { Specificity } \\
(\%)\end{array}$ & $\begin{array}{c}\text { False } \\
\text { negative } \\
(\%)\end{array}$ & $\begin{array}{c}\text { False } \\
\text { positive } \\
(\%)\end{array}$ & AUC & $\begin{array}{c}\text { Standard } \\
\text { error (AUC) }\end{array}$ & $\begin{array}{c}95 \% \mathrm{Cl} \text { for } \\
\text { AUC }\end{array}$ \\
\hline \multirow{3}{*}{ Planmeca } & $2 \mathrm{~mm}$ & 43.4 & 93.5 & 56.6 & 6.5 & 0.694 & 0.0250 & 0.632 to 0.750 \\
\hline & $3 \mathrm{~mm}$ & 50.7 & 93.5 & 49.3 & 6.5 & 0.726 & 0.0251 & 0.666 to 0.780 \\
\hline & $4 \mathrm{~mm}$ & 63.7 & 93.5 & 36.3 & 6.5 & 0.786 & 0.0243 & 0.730 to 0.836 \\
\hline \multirow{3}{*}{ Soredex } & $2 \mathrm{~mm}$ & 58.9 & 96.8 & 41.1 & 3.2 & 0.778 & 0.0236 & 0.721 to 0.828 \\
\hline & $3 \mathrm{~mm}$ & 62.7 & 96.8 & 37.3 & 3.2 & 0.802 & 0.0231 & 0.747 to 0.850 \\
\hline & $4 \mathrm{~mm}$ & 76.6 & 96.8 & 23.4 & 3.2 & 0.867 & 0.0207 & 0.818 to 0.907 \\
\hline \multirow{3}{*}{ NewTom } & $2 \mathrm{~mm}$ & 68.9 & 100 & 31.1 & 0.00 & 0.851 & 0.0206 & 0.800 to 0.893 \\
\hline & $3 \mathrm{~mm}$ & 74.2 & 100 & 25.8 & 0.0 & 0.871 & 0.0197 & 0.823 to 0.910 \\
\hline & $4 \mathrm{~mm}$ & 86.3 & 100 & 13.7 & 0.0 & 0.931 & 0.0155 & 0.893 to 0.960 \\
\hline \multirow{3}{*}{ PSP } & $2 \mathrm{~mm}$ & 4.8 & 98.3 & 95.2 & 1.7 & 0.516 & 0.0079 & 0.452 to 0.580 \\
\hline & $3 \mathrm{~mm}$ & 4.8 & 98.3 & 95.2 & 1.7 & 0.516 & 0.0079 & 0.452 to 0.580 \\
\hline & $4 \mathrm{~mm}$ & 7.2 & 98.3 & 92.8 & 1.7 & 0.528 & 0.0104 & 0.464 to 0.592 \\
\hline
\end{tabular}

AUC: Area under the curve; CI: Confidence interval

tion of all defects, irrespective of their depth, compared to periapical radiography. However, in terms of specificity, NewTom $3 \mathrm{G}$ showed the highest specificity followed by PSP digital periapical radiography, Soredex and Planmeca. Periapical radiography with the paralleling technique is commonly used for postoperative assessment following dental implant placement due to its simplicity, high resolution, low patient radiation dose and low cost. However, detection of dehiscence on periapical radiographs is difficult due to limitations such as 


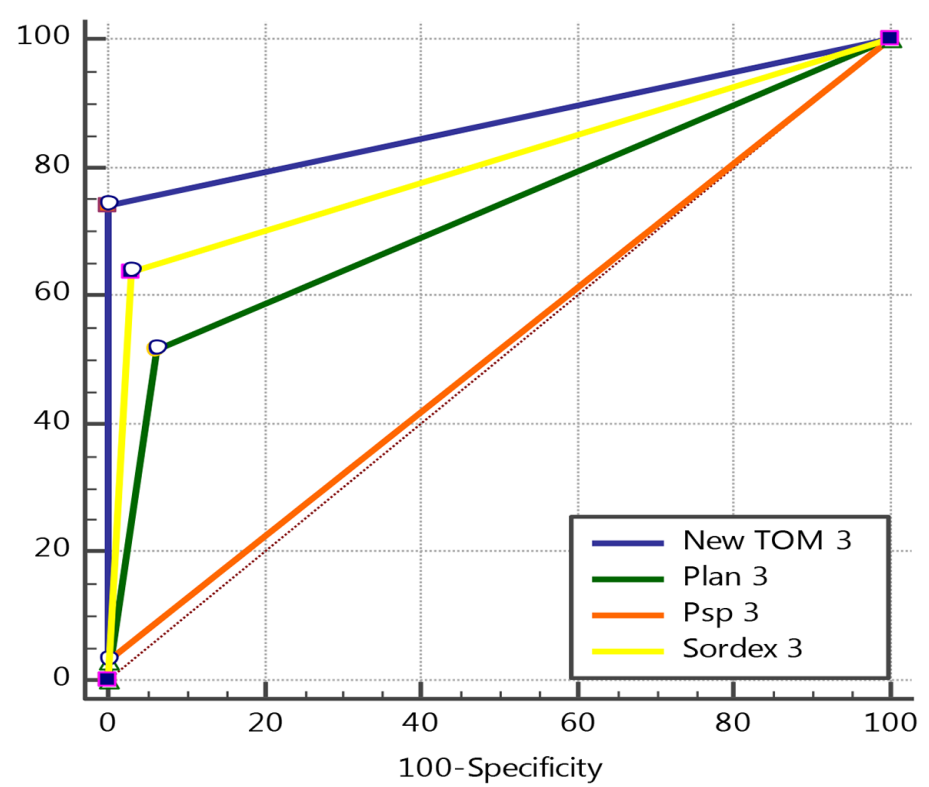

Figure 4: The receiver operating characteristic (ROC) curve for detection of $3 \mathrm{~mm}$ defects using the four imaging modalities.

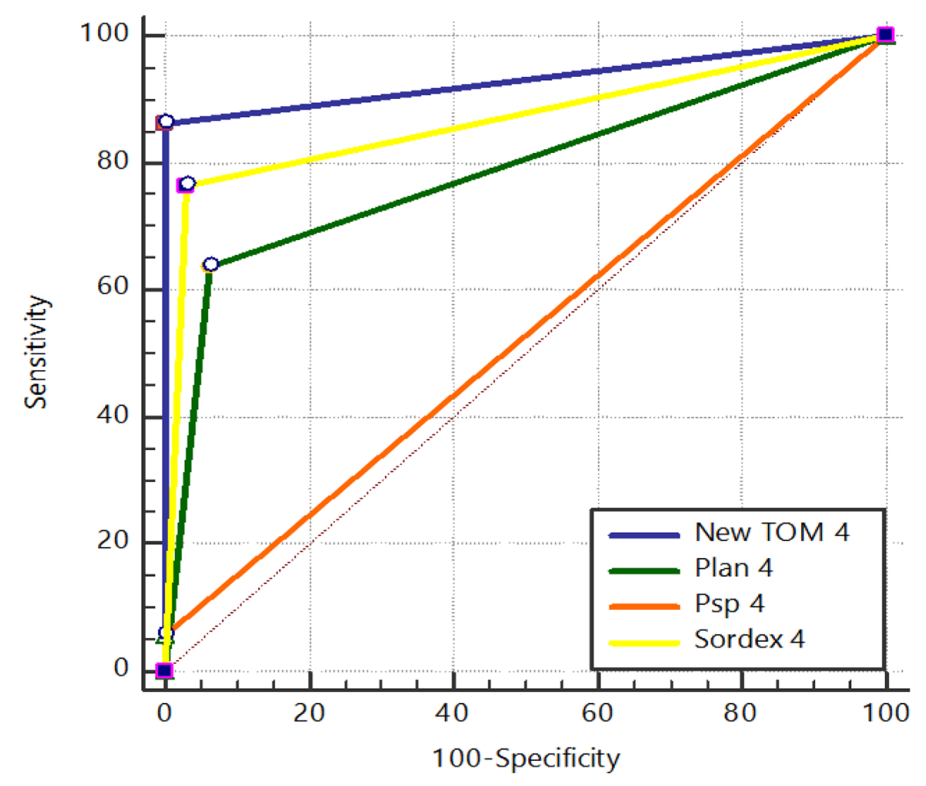

Figure 5: The receiver operating characteristic (ROC) curve for detection of $4 \mathrm{~mm}$ defects using the four imaging modalities.

superimposition of anatomical structures and provision of 2D views of 3D structures, making detection and assessment of the size of these defects extremely difficult on periapical radiographs. CBCT is a suitable modality for evaluation of buccal and lingual cortical plates and better visualization of the morphology of bone defects [14-16]. Bagis et al., [10] and Dehghani et al., [17] compared the diagnostic accuracy of CBCT with periapical radiography for detection of tunnel, fenestration and dehiscence bone defects and showed higher 
diagnostic accuracy of CBCT than periapical radiography. Also, they demonstrated that the diagnostic accuracy of CBCT increased with an increase in size of defects, which was in agreement with our findings.

Patel et al., [18] compared the diagnostic accuracy of periapical radiography and CBCT for detection of root resorption defects while Stavropoulous et al., [19] compared the diagnostic accuracy of periapical radiography and CBCT for detection of periapical lesions in pig jaws. Both studies showed that despite the differences in size and nature of lesions evaluated, the sensitivity of intraoral radiography was less than that of CBCT, irrespective of the size of lesions. ROC curve analysis also revealed that although periapical radiography had acceptable diagnostic accuracy, it was still lower than that of CBCT. However, in the study by Stavropoulous et al., [19] the specificity of periapical radiography was equal to that of CBCT. Similarly, in our study, the specificity of periapical radiography was ranked second after that of NewTom $3 \mathrm{G}$ and was higher than the specificity of Cranex and Planmeca CBCT systems. In our study, the sensitivity of NewTom 3G CBCT system was significantly higher than that of periapical radiography for detection of 2, 3 and $4 \mathrm{~mm}$ defects. Also, NewTom $3 \mathrm{G}$ showed lower false negative and false positive results in detection of defects, irrespective of their size. Takeshita et al., [20] compared periapical radiography and CBCT for detection and quantification of bone loss and indicated that the diagnostic accuracy of CBCT and the accuracy of measurements made on CBCT scans were both higher than periapical radiography, which was in agreement with our results.

In our study, NewTom $3 \mathrm{G}$ had the highest sensitivity followed by Soredex and Planmeca among the CBCT systems. Moreover, NewTom $3 \mathrm{G}$ showed the greatest value for the area under the ROC curve followed by Soredex and Planmeca; these findings highlight the superior diagnostic accuracy of NewTom $3 \mathrm{G}$ for all defects, irrespective of their size. Although the Planmeca CBCT system had relatively high specificity, indicating its acceptable efficacy for detection of sound cases, its maximum sensitivity was $63.7 \%$ (in detection of 4 $\mathrm{mm}$ defects), which was acceptable but still lower than that of NewTom $3 \mathrm{G}$ and Soredex. In our study, complete inter-observer agreement between the two observers at both first and second assessments for detection of sound samples. For detection of defected samples, the inter-observer agreement increased by an increase in depth of defects.

Saati et al., [21] compared the diagnostic accuracy of NewTom 3G, Soredex and Planmeca for detection of anatomical landmarks of dry human mandibles. In contrast to our results, they showed that Cranex 3D had the highest diagnostic accuracy followed by Planmeca and NewTom 3G. In their study, similar to ours, the voltage $(\mathrm{kVp})$ of NewTom $3 \mathrm{G} \mathrm{CBCT}$ system was higher than that of other systems, which would result in greater beam scattering and noise and eventually more difficult detection of anatomical landmarks especially finer structures. Kasraei et al., [22] compared the diagnostic accuracy of several imaging modalities for detection of recurrent caries under composite restorations. Contrary to our findings, they demonstrated that Cranex 3D was superior to NewTom $3 \mathrm{G}$ for this purpose. Difference between our findings and the results of the above-mentioned studies may be attributed to the absence of metal structures (which would create artifact) in the afore-mentioned two studies since metal artifacts can significantly compromise the diagnostic accuracy of imaging systems. Moreover, smaller voxel size in Cranex 3D and different type of detector (CMOS in Cranex 3D versus II/CCD in NewTom 3G) can also explain the higher diagnostic accuracy and resolution of Cranex 3D especially for detection of finer structures.

Van Dessel et al., [23] compared micro-CT as the gold standard and seven CBCT devices such as NewTom 3G, Soredex and Planmeca 
for structural analysis of alveolar bone by the use of morphometric indices and indicated that the afore-mentioned three CBCT devices had an acceptable accuracy comparable to that of micro-CT and almost similar to another one. On the contrary, our results revealed higher diagnostic accuracy of NewTom 3G. This controversy may be due to the similarity of exposure settings and particularly similar voltage $(\mathrm{kVp})$ of the three CBCT systems in their study [23].

The CBCT image quality is influenced by different types of artifacts, noise, lower contrast of soft tissue than hard tissue and difference in diagnostic value of different CBCT systems, which is due to the differences in type of detector and voxel size. Defects/lesions around dental implants create a radiolucent radiographic appearance, which resembles the beam hardening artifact, making the diagnosis more difficult. Metal artifacts caused by metal restorations and crowns further decrease the sensitivity of systems. In terms of type of detector, II/CCD has lower dynamic range, contrast and spatial resolution and higher noise and artifact than flat panel detector (FPD). Moreover, smaller voxel size yields higher resolution. Planmeca and Cranex 3D (in contrast to NewTom 3G) use FPD for image production and are expected to have higher diagnostic accuracy; however, our results showed that the exposure settings applied and limitation in increasing the voltage (kVP) in these systems resulted in their lower diagnostic accuracy than NewTom $3 \mathrm{G}$, which has a higher voltage (kVP). This finding highlights the more effective role of voltage $(\mathrm{kVp})$ in decreasing metal artifacts due to the presence of dental implant compared to other factors such as the type of detector and voxel size.

This study had an in vitro design and was conducted under controlled conditions. Teeth with metal restorations or crowns, which are commonly present in dental arch were not present in this study.

Mechanically induced artificial defects of- ten have a smooth and distinct border. Thus, future studies are required to chemically create defects using acid to create defects with irregular borders and better simulate the clinical setting. Also, future studies on the accuracy of imaging modalities for detection of anatomical landmarks and caries must be designed such that they include metal restorations and crowns to better simulate the clinical setting and to obtain more reliable results with higher generalizability to the clinical conditions.

\section{Conclusion}

In general, the inter-observer agreement in our study increased by an increase in depth of defects. CBCT systems were more efficient than periapical radiography for correct detection of presence/absence of crestal bone defects around dental implants. Among the three CBCT systems evaluated in this study, NewTom $3 \mathrm{G}$ had the highest diagnostic accuracy for detection of crestal bone defects.

\section{Acknowledgment}

We would like to thank the Hamadan University of Medical Sciences which provided invaluable facilities during this research and especial thanks to my research colleagues for their constant encouragement.

\section{Conflict of Interest}

None

\section{References}

1. Juodzbalys G, Kubilius M. Clinical and Radiological Classification of the Jawbone Anatomy in Endosseous Dental Implant Treatment. J Oral Maxillofac Res. 2013;4(2):e2. doi: 10.5037/jomr.2013.4202. PubMed PMID: 24422030. PubMed PMCID: PMC3886111.

2. De-Azevedo-V SL, Vaconcelos KD. Detection of periimplant fenestration and dehiscence with the use of two scan modes and the smallest voxel sizes of cone-beam computed tomography device. Oral Surg Oral Med Oral Pathol Oral Radiol. 2013;115(11):1216. doi: 10.1016/j.0000.2012.10.003. PubMed PMID: 23217543.

3. Jones $A A$, Cochran DL. Consequences of implant design. Dent Clin North Am. 2006;50:339-60. doi: 10.1016/j.cden.2006.03.008. PubMed PMID: 16818019. 
4. Eskandarloo A, Saati S, Purabdolahi Ardakani M, Jamalpour M, Mezerji N, Akheshteh V. Diagnostic accuracy of three cone beam computed tomography systems and periapical radiography for detection of fenestration around dental implants. Contemp Clin Dent. 2018;9(3)4:376-81. doi: 10.4103/ccd. ccd_103_18. PubMed PMID: 30166830. PubMed PMCID: PMC6104358.

5. Winkler S, Morris HF, Spray JR. Stability of implants and natural teeth as determined by the Periotest over 60 months of function. J Oral Implantol. 2001;27:198203. doi: 10.1563/1548-1336(2001)027<0198:SOIAN T>2.3.C0;2. PubMed PMID: 12500879.

6. King GN, Hermann JS, Schoolfield JD, Buser D, Cochran DL. Influence of the size of the microgap on crestal bone levels in non-submerged dental implants: a radiographic study in the canine mandible. J Periodontol. 2002;73:1111-17. doi: 10.1902/ jop.2002.73.10.1111. PubMed PMID: 12416767.

7. Chung WE, Rubenstein JE, Phillips KM, Raigrodski AJ. Outcomes assessment of patients treated with osseointegrated dental implants at the University of Washington Graduate Prosthodontic Program, 1998 to 2000. Int J Oral Maxillofac Implants. 2009;24(5):92735. PubMed PMID: 19865634.

8. Smith DE, Zarb GA. Criteria for success of osseointegrated endosseous implants. J Prosthet Dent. 1989;62:567-72. doi: 10.1016/0022-3913(89)900814. PubMed PMID: 2691661.

9. Kamburgolu K, Gulsahi A, Genç Y, Paksoy CS. A comparison of peripheral marginal bone loss at dental implants measured with conventional intraoral film and digitized radiographs. J Oral Implantol. 2012;38:21119. doi: 10.1563/AAID-JOI-D-09-00147. PubMed PMID: 20712442.

10. Bagis N, Kolsuz ME. Comparison of intraoral radiography and cone-beam computed tomography for the detection of periodontal defects: an in vitro study. BMC Oral Health. 2015;15(64):1-8. doi: 10.1186/s12903015-0046-2. PubMed PMID: 26016804. PubMed PMCID: PMC4446848.

11. Eskandarloo A, Arabi R, Bidgoli M, Yousefi F, Poorolajal J. Association between Marginal Bone Loss and Bone Quality at Dental Implant Sites Based on Evidence from Cone Beam Computed Tomography and Periapical Radiographs. Contemp Clin Dent. 2019;10(1):36-41. doi: 10.4103/ccd.ccd_185_18. PubMed PMID: 32015639. PubMed PMCID: PMC6974999.

12. Razavi T, Palmer RM, Davies J. Accuracy of measuring the cortical bone thickness adjacent to dental implants using cone beam computed tomography. Clin Oral Impl. 2010;21(5):718-25. doi: 10.1111/j.16000501.2009.01905.x. PubMed PMID: 20636726.

13. Shokri A, Eskandarloo A, Norouzi M, Majidi G, Aliyaly $A$. Diagnostic accuracy of cone-beam computed tomography scans with high- and low-resolution modes for the detection of root perforations. Imaging Sci Dent. 2018;48(1):11-9. doi: 10.5624/ isd.2018.48.1.11. PubMed PMID: 29581945. PubMed PMCID: PMC5863015.

14. De Smet E, Jacobs R, Gijbels F, Naert I. The accuracy and reliability of radiographic methods for the assessment of marginal bone level around oral implants. Dentomaxillofac Radiol. 2002;31(3):176-81. doi: 10.1038/ sj/dmfr/4600694. PubMed PMID: 12058265.

15. Wakoh M, Harada T, Otonari T, Otonari-Yamamoto $M$, Ohkubo $M$, Kousuge $Y$, et al. Reliability of linear distance measurement for dental implant length with standardized periapical radiographs. Bull Tokyo Dent Coll. 2006;47(3):105-15. doi: 10.2209/tdcpublication.47.105. PubMed PMID: 17344618.

16. Shokri A, Jamalpour M, Eskandarloo A, Godiny M, Amini P, Khavid A. Performance of Cone Beam Computed Tomography Systems in Visualizing the Cortical Plate in 3D Image Reconstruction: An In Vitro Study. Open Dent J. 2018;12:586-95. doi: 10.2174/1874210601812010586. PubMed PMID: 30288182. PubMed PMCID: PMC6142658.

17. Dehghani M, Montazer Lotf Elahi H. Comparing the accuracy of cone beam computed tomography,digital intraoral radiography and conventional intraoral radiography in the measurement of periodontal bone defects. J Res Dentomaxillofac Sci. 2016;1(1):34-9. doi: 10.29252/jrdms.1.1.34.

18. Patel S, Kanagasingam S, Mannocci F. Cone beam computed tomography (CBCT) in endodontics. Dent Update. 2009;37(6):373-9. doi: 10.12968/ denu.2010.37.6.373. PubMed PMID: 20929151.

19. Stavropoulos A, Wenzel A. Accuracy of cone beam dental CT, intraoral digital and conventional film radiography for the detection of periapical lesions. An ex vivo study in pig jaws. Clin Oral Investig. 2007;11(1):1016. doi: 10.1007/s00784-006-0078-8. PubMed PMID: 17048029.

20. Takeshita WM, Iwaki LCV, Da Silva MC, Tonin RH. Evaluation of diagnostic accuracy of conventional and digital periapical radiography, panoramic radiography, and cone-beam computed tomography in the assessment of alveolar bone loss. Contemp Clin Dent. 2014;5(3):318-23. doi: 10.4103/0976237X.137930. PubMed PMID: 25191066. PubMed PMCID: PMC4147806.

21. Saati S, Kaveh F, Yarmohammadi SH. Comparison of Cone-Beam computed tomography and Multi slice computed tomography image quality of human dried mandible using 10 anatomical landmarks. J Clin Diagn Res. 2017;11(2):13-6. doi: 10.7860/ JCDR/2017/20637.9253. PubMed PMID: 28384972. PubMed PMCID: PMC5376866.

22. Kasraei SH, Shokri A, Poorolajal J, Khajeh S, Rahmani $H$. Comparison of cone-beam computed tomography and Intraoral radiography in detection of recurrent caries under composite restorations. Braz Dent J. 2017;28(1):85-91. doi: 10.1590/01036440201701248. PubMed PMID: 28301024.

23. Van Dessel J, Nicolielo L, Huang Y, Coudyzer W, Salmon B, Lambrichts I, Jacobs R. Accuracy and reliability of different cone-beam computed tomography(CBCT) devices for structural analysis of alveolar bone in comparison with multislice CT and Micro-ct. Eur $J$ Oral implantol. 2017;10(1):95-105. PubMed PMID: 28327698. 